Bull. Austral. Math. Soc.

VOL. 52 (1995) [97-105]

\title{
SMOOTH FAMILIES OF FIBRATIONS AND ANALYTIC SELECTIONS OF POLYNOMIAL HULLS
}

\author{
Miran Černe
}

\begin{abstract}
Constructed are strictly increasing smooth families $\Sigma^{t} \subseteq \partial D \times \mathbf{C}^{2}, t \in[0,1]$, of fibrations over the unit circle with strongly pseudoconvex fibers all diffeomorphic to the ball $\overline{B^{4}}$ such that there is no analytic selection of the polynomial hull of $\Sigma^{0}$ and which end at the product fibration $\Sigma^{1}=\partial D \times \overline{B^{4}}$. In particular these examples show that the continuity method for describing the polynomial hull of a fibration over $\partial D$ fails even if the complex geometry of the fibers is relatively simple.
\end{abstract}

\section{INTRODUCTION}

Let $\mathcal{P}_{n}$ be the algebra of holomorphic polynomials in $n$ complex variables and let $X \subseteq \mathbf{C}^{n}$ be a compact subset of the complex space $\mathbf{C}^{n}$. The polynomial hull $\widehat{X}$ of $X$ is defined as

$$
\widehat{X}:=\left\{z_{o} \in \mathbf{C}^{n} ;\left|p\left(z_{o}\right)\right| \leqslant \sup _{z \in X}|p(z)|, p \in \mathcal{P}_{n}\right\} .
$$

Let $D \subseteq \mathbf{C}$ be the unit disc in the complex plane $\mathbf{C}$ and let $\partial D$ be its boundary, the unit circle in C. An $H^{\infty}$ analytic disc with boundary in $X$ is an $H^{\infty}$ mapping $h: D \rightarrow \mathbf{C}^{n}$ such that

$$
h(\xi) \in X \text { almost everywhere } d m(\xi),
$$

where $d m(\xi)$ stands for the Lebesgue measure on $\partial D$. By the maximum principle it follows immediately that if $h$ is an $H^{\infty}$ analytic disc with boundary in $X$, then the whole disc $h(D)$ lies in the polynomial hull $\widehat{X}$ of $X$, that is, $h(D) \subseteq \widehat{X}$. It is a classical result by Stolzenberg, [10], that it is not always the case that the set $\widehat{X} \backslash X$ can be given as the union of the $H^{\infty}$ analytic discs with boundaries in $X$. Later Wermer, [11], refined Stolzenberg's example and constructed a fibration over the unit circle $\partial D$ with fibers in $\mathbf{C}$ with the same property.

Received 20th September, 1994

I would like to thank my thesis advisor prof. Franc Forstneric for many stimulating discussions, helpful suggestions and constant encouragment. This work was supported in part by a grant from the Ministry of Science and Technology of the Republic of Slovenia.

Copyright Clearance Centre, Inc. Serial-fee code: 0004-9729/95 \$A2.00+0.00. 
On the other hand there is a series of papers $[1,4,5,7,8,9]$ on the polynomial hull of a fibration

$$
X:=\bigcup_{\xi \in \partial D}\{\xi\} \times X_{\xi} \subseteq \partial D \times \mathbf{C}^{n}
$$

over $\partial D$, which show that in the case the geometry of the fibers $X_{\xi}, \xi \in \partial D$, is tame, that is, arbitrary dimension $n$ and all fibers are geometrically convex $[1,4,7,9]$ or $n=1$ and the fibers are only connected and simply connected $[5,8]$, one can describe the polynomial hull of $X$ as the union of the graphs $\{(z, h(z)) ; z \in D\}$ of the $H^{\infty}$ analytic discs $h$ in $\mathbf{C}^{n}$ for which

$$
h(\xi) \in X_{\xi} \text { almost everywhere } d m(\xi) \text {. }
$$

A disc $h$ of this kind is called an analytic selection of the polynomial hull of $X$. An example by Helton and Merino, [6], shows that the condition on the fibers to be only connected and simply connected is not enough for the same result to hold for $n \geqslant 2$. Namely, they found an example of a fibration $X$ over $\partial D$ with connected and simply connected fibers in $\mathbf{C}^{2}$, whose polynomial hull $\hat{X}$ is nontrivial, but there is no graph of an $H^{\infty}$ analytic disc whose boundary lies in $X$.

All proofs of the above positive results for $n \geqslant 2$ are essentially based on a very clever use of the Hanh-Banach theorem and are, therefore, linear (convex) in their nature. One could hope that exploiting the complex geometry of the fibers $X_{\xi}, \xi \in \partial D$, one could still get some positive results on the description of the polynomial hull of $X$ as Forstnerič did in [5] in the case of one dimensional fibers. See also [8]. In this paper we give two examples, inspired by the example by Helton and Merino, [6], which show that the so called continuity method for describing the polynomial hull of a fibration over $\partial D$, which was so successfully used by Forstnerič for $n=1$, [5], fails even in the case the complex geometry of the fibers is simple. See also [2].

ThEOREM 1.1. There exists a smooth family of fibrations

$$
\Sigma^{t}:=\bigcup_{\xi \in \partial D}\{\xi\} \times \Sigma_{\xi}^{t}, \quad(t \in[0,1])
$$

in $\partial D \times \mathbf{C}^{2}$ with the following properties:

1. for all $t \in[0,1]$ and for all $\xi \in \partial D$ the interiors $\Omega_{\xi}^{t}$ of the fibers $\Sigma_{\xi}^{t}$ are strongly pseudoconvex domains in $\mathbf{C}^{2}$ with smooth boundaries, all diffeomorphic to the ball and such that $\overline{\Omega_{\xi}^{t}}=\Sigma_{\xi}^{t}$,

2. all fibers of the fibration $\Sigma^{1}$ are Euclidean balls in $\mathbf{C}^{2}$,

3. the family is strictly increasing in the sense that for all $\xi \in \partial D$ and for all pairs $t, \tau \in[0,1], t<\tau$, the inclusion

$$
\Sigma_{\xi}^{t} \subseteq \Omega_{\xi}^{r}
$$


holds,

4. the fibration $\Sigma^{0}$ has the property that its polynomial hull is nontrivial, but there is no $H^{\infty}$ analytic selection of the fibration $\Sigma^{0}$.

ThEOREM 1.2. There exists a smooth family of fibrations

$$
\Sigma^{t}:=\bigcup_{\xi \in \partial D}\{\xi\} \times \Sigma_{\xi}^{t}, \quad(t \in[0,1])
$$

in $\partial D \times \mathbf{C}^{2}$ with the properties (1), (2) and (3) of Theorem 1 and with the additional properties :

4. for every $t \in[0,1]$ and for every $\xi \in \partial D$ there is a fixed small open ball $B_{o}$ included in the interior $\Omega_{\xi}^{t}$ of all fibers $\Sigma_{\xi}^{t}$,

5. there is a point $z_{o}$ in the polynomial hull of $\Sigma^{0}, z_{o} \notin \Sigma^{0}$, through which there is no graph of an $H^{\infty}$ analytic selection of $\Sigma^{0}$.

\section{BLOWING UP AN ARC}

In this section we prove the following proposition.

\section{PROPOSITION 2.1.}

Let $\gamma$ be a smooth arc in $\mathbf{R}^{2} \subseteq \mathbf{C}^{2}$. Then there exists a smooth strictly plurisubharmonic function $\widetilde{\rho}$ on $\mathbf{C}^{2}$ such that

(a) $\gamma=\left\{z \in \mathbf{C}^{2} ; \tilde{\rho}(z)=0\right\}=\left\{z \in \mathbf{C}^{2} ; \nabla \tilde{\rho}(z)=0\right\}$ and

(b) there exists $C>0$ such that for every $c \geqslant C$ the level set $\left\{z \in \mathbf{C}^{2} ; \tilde{\rho}(z)=\right.$ c\} is an Euclidean 3-sphere.

Proof: Let $f$ be any smooth nonnegative function on $\mathbf{R}^{2}$ such that

(a) the zero set of $f$ and the zero set of the gradient $\nabla f$ are both equal to $\gamma$ and

(b) there exists an $r_{o}>0$ such that $f\left(x_{1}, x_{2}\right)=x_{1}^{2}+x_{2}^{2}$ for $x_{1}^{2}+x_{2}^{2} \geqslant r_{o}^{2}$.

Here the coordinates in $\mathbf{R}^{2} \subseteq \mathbf{C}^{2}$ are $x_{1}, x_{2}$ and the coordinates in $\mathbf{C}^{2}$ are $z_{1}=x_{1}+i y_{1}$ and $z_{2}=x_{2}+i y_{2}$. For $\lambda>0$ we define

$$
\rho_{\lambda}\left(z_{1}, z_{2}\right)=f\left(x_{1}, x_{2}\right)+\lambda\left(y_{1}^{2}+y_{2}^{2}\right) .
$$

Then

(1) the zero set of $\rho_{\lambda}$ and the zero set of $\nabla \rho_{\lambda}$ are both equal to the arc $\gamma$ and

(2) the Levi form of the function $\rho_{\lambda}$ is

$$
L\left(\rho_{\lambda}\right):=\frac{1}{4}\left(\begin{array}{cc}
f_{x_{1} x_{1}}+2 \lambda & f_{x_{1} x_{2}} \\
f_{x_{1} x_{2}} & f_{x_{2} x_{2}}+2 \lambda
\end{array}\right)
$$


where the notation $f_{x_{i} x_{j}}$ stands for the second partial derivative of the function $f$ with respect to $x_{i}$ and $x_{j}, i, j=1,2$.

Condition (b) on the function $f$ ensures that if $\lambda$ is large enough, the function $\rho_{\lambda}$ is strictly plurisubharmonic on $\mathbb{C}^{2}$. We fix such a $\lambda$ and denote the function $\rho_{\lambda}$ by $\rho$.

Let $\chi: \mathbf{R} \rightarrow[0,1]$ be a smooth function whose support is contained in the interval $\left[-1,\left(r_{o}+2\right)^{2}\right]$ and which equals 1 on the closed interval $\left[0,\left(r_{o}+1\right)^{2}\right]$. Also, let $g$ be a smooth nonnegative function on $\mathbf{R}$ such that

(1) $g(x)=0$ for $x \leqslant r_{o}^{2}$,

(2) $g^{\prime}(x)>0$ and $g^{\prime \prime}(x) \geqslant 0$ for $x>r_{0}^{2}$,

(3) $\rho(z) \chi^{\prime}\left(|z|^{2}\right)+g^{\prime}\left(|z|^{2}\right) \geqslant 0$ for every $z \in \mathbf{C}^{2}$.

For $\varepsilon \in(0,1)$ we define

$$
\tilde{\rho}_{e}(z)=\varepsilon \chi\left(|z|^{2}\right) \rho(z)+g\left(|z|^{2}\right) \quad\left(z \in \mathbf{C}^{2}\right) .
$$

If $\varepsilon$ is small enough, the function $\tilde{\rho}$ is strictly plurisubharmonic on $\mathbf{C}^{2}$ and its zero set is the arc $\gamma$. We fix such an $\varepsilon$ and denote the corresponding function by $\tilde{\rho}$. Thus the prcposition will be proved once we prove the following lemma.

LEMma 2.1. The zero set of the gradient $\nabla \widetilde{\rho}$ is the arc $\gamma$.

Proof: Let $z^{o}=\left(x_{1}^{o}+i y_{1}^{o}, x_{2}^{o}+i y_{2}^{o}\right)$ be a point where the gradient $\nabla \widetilde{\rho}$ is zero. We consider the following three cases :

1. Case $\left|z^{\circ}\right|<r_{0}$. Then $\tilde{\rho}=\varepsilon \rho$ in a neighbourhood of the point $z^{\circ}$ and thus $z^{\circ} \in \gamma$.

2. Case $\left|z^{o}\right|>r_{o}+2$. Then $\tilde{\rho}(z)=g\left(|z|^{2}\right)$ in a neighbourhood of the point $z^{o}$. Since $g^{\prime}(x)>0$ for $x>r_{0}^{2}$, we get a contradiction.

3. Case $r_{o} \leqslant\left|z^{\circ}\right| \leqslant r_{o}+2$. The $y$ components of the gradient $\nabla \tilde{\rho}$, that is, the derivatives of $\tilde{\rho}$ with respect to $y_{1}$ and $y_{2}$ at the point $z$ are equal to

$$
\frac{\partial \widetilde{\rho}}{\partial y_{j}}(z)=2\left(\lambda \varepsilon \chi\left(|z|^{2}\right)+\varepsilon \rho(z) \chi^{\prime}\left(|z|^{2}\right)+g^{\prime}\left(|z|^{2}\right)\right) y_{j} \quad(j=1,2) .
$$

Therefore, if $\nabla \tilde{\rho}\left(z^{\circ}\right)=0$, one concludes that since

$$
\lambda \varepsilon \chi\left(|z|^{2}\right)+\varepsilon \rho(z) \chi^{\prime}\left(|z|^{2}\right)+g^{\prime}\left(|z|^{2}\right)>\varepsilon\left(\rho(z) \chi^{\prime}\left(|z|^{2}\right)+g^{\prime}\left(|z|^{2}\right)\right) \geqslant 0
$$

on $\mathbf{C}^{2}$, it follows

$$
y_{1}^{\circ}=y_{2}^{o}=0 .
$$

Our initial assumption (b) on the function $f$ and the fact that $\left|z^{\circ}\right| \geqslant r_{0}$ imply

$$
f_{x_{1}}\left(x_{1}^{o}, x_{2}^{\circ}\right)=2 x_{1}^{o} \quad \text { and } f_{x_{2}}\left(x_{1}^{o}, x_{2}^{o}\right)=2 x_{2}^{o} .
$$


The $x$ components, that is, the derivatives with respect to $x_{1}$ and $x_{2}$ variables, of the equation $\nabla \tilde{\rho}\left(z^{\circ}\right)=0$, together with (1) give

$$
x_{1}^{0}=x_{2}^{o}=0 .
$$

Hence also the assumption $r_{0} \leqslant\left|z^{\circ}\right| \leqslant r_{0}+2$ leads to a contradiction and the lemma, thus also the proposition, is proved.

A more geometric interpretation of the above proposition is that for every simple arc $\gamma$ in $\mathbf{R}^{2} \subseteq \mathbf{C}^{2}$ there exists a smooth family of strictly pseudoconvex domains

$$
\Omega_{t}:=\left\{z \in \mathbf{C}^{2} ; \tilde{\rho}(z)<t\right\} \quad(t \in(0, \infty)),
$$

in $\mathbf{C}^{2}$ with smooth boundary which starts at $\gamma$, is strictly increasing in the sense that for each pair of parameters $t<\tau$ the domain $\Omega_{t}$ is compactly included in the domain $\Omega_{\tau}$ and which ends at some large Euclidean ball. Observe also that since the gradient $\nabla \tilde{\rho}$ is nonzero except on $\gamma$ all the domains $\Omega_{t}, t \in(0, \infty)$, are topological cells.

REMARK 2.1. If one is given a smooth family of simple arcs $\gamma_{\xi}, \xi \in \partial D$, in $\mathbf{R}^{2} \subseteq \mathbf{C}^{2}$, then one can choose a smooth family of smooth functions $f_{\xi}, \xi \in \partial D$, satisfying conditions (a) and (b) for each $\xi \in \partial D$. Since the set of parameters is compact, the functions $\chi$ and $g$ and the constants $\lambda$ and $\varepsilon$ can be chosen uniformly, that is, independent of the parameter $\xi \in \partial D$, and the corresponding strictly plurisubharmonic functions $\tilde{\rho}_{\xi}(z)$ vary smoothly in both variables $\xi$ and $z$.

REMarK 2.2. The above construction can be applied to any arc $\gamma$ in $\mathbf{C}^{2}$ for which there exists a holomorphic automorphism $\Phi$ of $\mathbf{C}^{2}$ such that $\Phi(\gamma) \subseteq \mathbf{R}^{2}$.

\section{First family of fibrations}

We consider now the following family of arcs in $\mathbf{R}^{2} \subseteq \mathbf{C}^{2}$. Let $\gamma_{1}$ be the semicircle in $\mathbf{R}^{2}$ given by the equation

$$
x_{1}^{2}+x_{2}^{2}=1, \quad x_{2} \geqslant 0 .
$$

For $\xi \in \partial D$ we denote by $R_{\xi}$ the map

$$
R_{\xi}: \mathrm{C}^{2} \longrightarrow \mathrm{C}^{2}
$$

defined by

$$
R_{\xi}\left(z_{1}, z_{2}\right):=\left(\xi z_{1}, z_{2}\right) .
$$

Observe that $R_{\xi}$ is a linear isomorphism of $\mathbf{C}^{2}$. For $\xi \in \partial D$ such that $0 \leqslant \arg (\xi) \leqslant \pi / 2$ or $(3 \pi) / 2 \leqslant \arg (\xi) \leqslant 2 \pi$ let

$$
\gamma_{\xi}:=\gamma_{1}
$$


and for the parameters $\xi \in \partial D$ such that $\pi / 2<\arg (\xi)<(3 \pi) / 2$ we smoothly perturb the initial arc $\gamma_{1}$ to get arcs $\gamma_{\xi}$ which do not pass through the point $(0,1)$ but they still pass through the points $(1,0)$ and $(-1,0)$. For instance, for $\xi=e^{i s}$ one may take $\gamma_{\xi}$ to be defined by the equation

$$
(1-\varrho(s))^{2} x_{1}^{2}+x_{2}^{2}=(1-\varrho(s))^{2}, \quad x_{2} \geqslant 0,
$$

where $\varrho: \mathbf{R} \rightarrow[0,1)$ is any smooth function whose support is the interval $[\pi / 2,(3 \pi) / 2]$. We define

$$
\tilde{\gamma}_{\xi}:=R_{\sqrt{\xi}}\left(\gamma_{\xi}\right)
$$

Here by $\sqrt{\xi}$ we mean the principal branch of the square root, that is, $\sqrt{-1}=i$. Since we have $\gamma_{\xi}=\gamma_{1}$ in a neighbourhood of $\xi=1$ and since the arc $\gamma_{1}$ is symmetric with respect to the $x_{2}$-axis, the family of arcs $\widetilde{\gamma}_{\xi}, \xi \in \partial D$, is smooth. Using our initial construction for an arc $\gamma \subseteq \mathbf{R}^{2}$ and Remarks 2.1 and 2.2, one gets a smooth family of fibrations $\Sigma^{t}, t>0$, in $\partial D \times \mathbf{C}^{2}$ such that for each $t$ the interiors of all fibers are strongly pseudoconvex domains with smooth boundaries and for $t$ large enough all fibers of $\Sigma^{t}$ are Euclidean balls centred at the point $(0,0)$ with the fixed radius $\sqrt{t}$. Also, for every pair $t, \tau \in(0, \infty), t<\tau$, all fibers of the fibration $\Sigma^{t}$ are included in the interiors of the corresponding fibers of $\Sigma^{\tau}$.

REMARK 3.1. Observe that by a theorem of Docquier and Grauert [3] the above properties of the family of fibrations $\Sigma^{t}, t>0$, assure that the fibers of $\Sigma^{t}$ remain polynomially convex for each parameter $t>0$.

To finish our example we first observe that since

$$
(\sqrt{\xi}, 0),(-\sqrt{\xi}, 0) \in \tilde{\gamma}_{\xi} \quad(\xi \in \partial D),
$$

the polynomial hull of $\Sigma^{t}$ contains the point $(0,0,0)$ for all $t>0$. Finally we prove the following lemma.

Lemma 3.1. For $t>0$ small enough there is no graph of an $H^{\infty}$ analytic mapping $F: D \rightarrow \mathbf{C}^{2}$ with boundary in the fibration $\Sigma^{t} \subseteq \partial D \times \mathbf{C}^{2}$.

Proof: We prove the lemma for

$$
\Sigma^{0}:=\bigcup_{\xi \in \partial D}\{\xi\} \times \tilde{\gamma}_{\xi}
$$

Once this is proved the normal family argument finishes the proof of the lemma. Namely, assume that there is a sequence $t_{n} \downarrow 0, n \in \mathrm{N}$, such that for all $n$ there exists an $H^{\infty}$ analytic selection $F_{n}$ for $\Sigma^{n}:=\Sigma^{t_{n}}$. By the normal family argument there exists a 
subsequence of $\left\{F_{n}\right\}_{n \in N}$, still denoted by $F_{n}$, which normally converges to an $H^{\infty}$ function $F_{o}$. Then for every holomorphic polynomial $p$ in three variables and every $z \in D$ we have

$$
\left|p\left(z, F_{o}(z)\right)\right|=\lim _{n}\left|p\left(z, F_{n}(z)\right)\right| \leqslant \lim _{n} \sup _{x \in \Sigma^{n}}|p(x)|=\sup _{x \in \Sigma^{0}}|p(x)|
$$

The inequality follows because the discs $F_{n}, n \in \mathrm{N}$, are analytic selections for the fibrations $\Sigma^{n}, n \in \mathbf{N}$, and the last equality is true since the family of fibrations $\Sigma^{t}$, $t \geqslant 0$, is continuous in Hausdorff topology of compact sets in $\mathbf{C}^{2}$. Therefore the graph $\left\{\left(z, F_{\mathrm{o}}(z)\right) ; z \in D\right\}$ is contained in the polynomial hull of $\Sigma^{0}$ and so $F_{\mathrm{o}}$ is an analytic selection of $\Sigma^{0}$. Here we used the fact that all fibers of the fibration $\Sigma^{0}$ are polynomialy convex in $\mathbf{C}^{2}$.

Let us assume now that there is an analytic mapping

$$
(f, g): D \longrightarrow \mathbf{C}^{2}
$$

such that

$$
(f(\xi), g(\xi)) \in \widetilde{\gamma}_{\xi} \quad(\text { almost everywhere } \xi \in \partial D)
$$

Therefore the imaginary part of the function $g$ almost everywhere on $\partial D$ equals to 0 and thus $g$ is a constant function, that is, there is a real number $a \in[0,1]$ such that $g(\xi)=a$ for every $\xi \in \partial D$. Since the arcs $\tilde{\gamma}_{\xi}$ for $\pi / 2<\arg (\xi)<(3 \pi) / 2$ do not pass through the point $(0,1)$ the constant $a$ has to be less than 1 . But then for all $\xi \in \partial D$ we have

$$
((1 / \sqrt{\xi}) f(\xi), a) \in \gamma_{\xi}
$$

and so

$$
f(\xi)^{2}=\left(1-a^{2}\right) \xi \text { almost everywhere } d m(\xi),
$$

which leads to a contradiction.

\section{SECOND FAMILY OF FIBRATIONS}

Let $\gamma \subseteq \mathbf{R}^{2} \subseteq \mathbf{C}^{2}$ be the arc

$$
x_{1}^{2}+x_{2}^{2}=1, \quad x_{2} \geqslant 0
$$

as before. Let $X_{1}:=\gamma$ and let

$$
X_{\xi}:=R \sqrt{\xi} X_{1}
$$

Since again

$$
(\sqrt{\xi}, 0),(-\sqrt{\xi}, 0) \in X_{\xi}(\xi \in \partial D)
$$


it is obvious that the polynomial hull of

$$
X:=\bigcup_{\xi \in O D}\{\xi\} \times X_{\xi}
$$

contains the point $(0,0,0)$.

Lemma 4.1. There is no $B^{\infty}$ analytic selection $F: D \rightarrow \mathrm{C}^{2}$ of $X$ which passes through the point $(0,0)$.

Proof: Let us assume that there is an analytic disc $F=(f, g)$ whose graph has boundary almost everywhere contained in $X$ and is such that $F(0)=(0,0)$. This implies, as in the previous section, that

$$
g(\xi)=0 \quad(\xi \in \bar{D}) .
$$

Thus

$$
\left.f^{2}(\xi)=\xi \quad \text { (almost everywhere } \xi \in \partial D\right)
$$

a contradiction.

Since all fibers $X_{\xi}, \xi \in \partial D$, of $X$ contain the point $(0,1)$, all fibers of the fibrations $\Sigma^{t}, t>0$, constructed similarly as the first family of fibrations, have the point $(0,1)$ in its interior. Finally, repeating the argument from the previous section shows that there exists $t_{o}>0$ such that there is no analytic selection for the fibration $\Sigma^{t_{o}}$ which passes through the point $(0,0)$. Details are omitted.

\section{REFERENCES}

[1] H. Alexander and J. Wermer, 'Polynomial hulls with convex fibers', Math. Ann. 271 (1985), 99-109.

[2] M. Cerne, Analytic discs with boundaries in a generating CR-manifold, Ph.D. Thesis (Madison, 1994).

[3] F. Docquier and H. Grauert, 'Levisches Problem and Rungescher Satz für Teilgebiete Steinscher Mannigfaltigkeiten', Math. Ann. 140 (1960), 94-123.

[4] F. Forstnerič, 'Polynomially convex hulls with piecewise smooth boundaries', Math. Ann. 276 (1986), 97-104.

[5] F. Forstnerič, 'Polynomial hulls of sets fibered over the circle', Indiana Univ. Math. J. 37 (1988), 869-889.

[6] J.W. Helton and O. Merino, 'A fibered polynomial hull without an analytic selection', Michigen Math. J. 41 (1994), 285-287.

[7] Z. Slodkowski, 'Polynomial hulls with convex sections and interpolating spaces', Proc. Amer. Math. Soc. 96 (1986), 255-260.

[8] Z. Slodkowski, 'Polynomial hulls in $\mathbf{C}^{2}$ and quasicircles', Ann. Scuola Norm. Sup. Pisa Cl. Sci. 16 (1989), 367-391. 
[9] Z. Slodkowski, 'Polynomial hulls with convex fibers and complex geodesics', J. Funct. Anal. 94 (1990), 156-176.

[10] G. Stolzenberg, 'A hull with no analytic structure', J. Math. Mech. 12 (1963), 103-111.

[11] J. Wermer, 'Polynomial convex hulls and analyticity', Ark. Mat. 20 (1982), 129-13.

University of Ljubljans, IMFM

Jadranska 19

61111 Ljubljana

Slovenia 